\title{
Alteration of Metabolic Profile and Potential Biomarkers in the Plasma of Alzheimer's Disease
}

\author{
Yaping Shao, ${ }^{1,2}$, Yang Ouyang3,4, Tianbai Li, ${ }^{1,2}$ Xinyao Liu ${ }^{1,2}$, Xiaojiao Xu1, ${ }^{1,}$, Song Li, ${ }^{1,}$, \\ Guowang $\mathrm{Xu}^{3 *}$, Weidong $\mathrm{Le}^{1,2^{*}}$ \\ ${ }^{1}$ Center for Clinical Research on Neurological Diseases, The First Affiliated Hospital, Dalian Medical University, \\ Dalian, China. ${ }^{2}$ Liaoning Provincial Key Laboratory for Research on the Pathogenic Mechanisms of Neurological \\ Diseases, The First Affiliated Hospital, Dalian Medical University, Dalian, China. ${ }^{3}$ CAS Key Laboratory of \\ Separation Science for Analytical Chemistry, Dalian Institute of Chemical Physics, Chinese Academy of Sciences, \\ Dalian, China. ${ }^{4}$ University of Chinese Academy of Sciences, Beijing, China
}

[Received October 22, 2019; Revised January 24, 2020; Accepted February 17, 2020]

\begin{abstract}
The expending of elderly population worldwide has resulted in a dramatic rise in the incidence of chronic diseases such as Alzheimer's disease (AD). Inadequate understanding of the mechanisms underlying AD has hampered the development of efficient tools for definitive diagnosis and curative interventions. Previous studies have attempted to discover reliable biomarkers of $\mathrm{AD}$, but these biomarkers can only be measured through invasive (neuropathological markers in cerebrospinal fluid) or expensive (positron emission tomography scanning or magnetic resonance imaging) techniques. Metabolomics is a high-throughput technology that can detect and catalog large numbers of small metabolites and may be a useful tool for characterization of AD and identification of biomarkers. In this study, we used ultra-performance liquid chromatography-mass spectrometry based untargeted metabolomics to measure the concentrations of plasma metabolites in a cohort of subjects with AD ( $n=44)$ and cognitively normal controls $(C t r l, n=94)$. The AD group showed marked reductions in levels of polyunsaturated fatty acids, acyl-carnitines, degradation products of tryptophan, and elevated levels of bile acids compared to the Ctrl group. We then validated the results using an independent cohort that included subjects with $\operatorname{AD}(n=30)$, mild cognitive impairment (MCI, $n=13)$, healthy controls $(n=43)$, and non-AD neurological disease controls (NDC, $n=31$ ). We identified five metabolites comprising cholic acid, chenodeoxycholic acid, allocholic acid, indolelactic acid, and tryptophan that were able to distinguish patients with AD from both Ctrl and NDC with satisfactory sensitivity and specificity. The concentrations of these metabolites were significantly correlated with disease severity. Our results also suggested that altered bile acid profiles in AD and MCI might indicate early risk for the development of AD. These findings may allow for development of new approaches for diagnosis of $\mathrm{AD}$ and may provide novel insights into AD pathogenesis.
\end{abstract}

Key words: Alzheimer's disease, metabolomics, biomarker, metabolic pathway alteration, plasma

Alzheimer's disease (AD) is a progressive neurodegenerative disorder that accounts for $60-80 \%$ of all dementia cases. Inadequate understanding of the etiology of $\mathrm{AD}$ and confounding factors that contribute to its genotypic and phenotypic heterogeneities may explain the lack of development of curative therapies [1,2]. Genetic causes account for only $1-5 \%$ of all AD cases, and $>95 \%$ of cases are sporadic [3]. Clinical diagnosis of $\mathrm{AD}$ is primarily reached based on medical history, patient symptoms, neuroimaging, and neuropsychological

*Correspondence should be addressed to: Dr. Weidong Le, Liaoning Provincial Center for Clinical Research on Neurological Diseases, the First Affiliated Hospital, Dalian Medical University, Dalian, China. Email: wdle@sibs.ac.cn and Dr. Guowang Xu, Dalian Institute of Chemical Physics, Chinese Academy of Sciences, Dalian, China. Email: xugw@ dicp.ac.cn.

Copyright: () 2020 Shao Y et al. This is an open-access article distributed under the terms of the Creative Commons Attribution License, which permits unrestricted use, distribution, and reproduction in any medium, provided the original author and source are credited. 
evaluation, and typically occurs too late for effective disease modification [4]. Although several methods have been developed for diagnosis of $\mathrm{AD}$, including quantification of amyloid- $\beta(A \beta)$ and tau protein levels in cerebrospinal fluid, measurement of $A \beta$ using positronemission tomography imaging, and analysis of atrophy using magnetic resonance imaging, these procedures are either invasive (lumbar puncture) or expensive (imaging) [5-7]. Therefore, identification of peripheral bloodderived biomarkers capable of identifying $\mathrm{AD}$ during early stages is an urgent priority.

Numerous studies have identified blood biomarkers of $\mathrm{AD}$, the majority of which were derived from known disease markers such as $A \beta$ and tau, which represents narrow hypothesis-driven biomarker discovery [8, 9]. Although many studies have proposed several biomarker candidates such as inflammation or oxidative stressrelated proteins, cytokines, chemokines, growth factors, and lipids, there is still a clear need for a broader study of biomarker that could facilitate better understanding of the disease mechanisms [6, 10, 11]. Identification of novel biological characteristics of $\mathrm{AD}$ could contribute to the discovery of novel circulating biomarkers associated with risk for development of $\mathrm{AD}$, and identification of therapeutic targets.

Metabolomics is a rapidly expanding field in systems biology used to explore the molecular basis of biological aspects of diseases [12]. Increasing numbers of studies have shown that dysregulation of various metabolic pathways, such as cholesterol metabolism and energy metabolism, may contribute to onset of $\mathrm{AD}$ pathology and AD-associated cognitive impairment [13]. Characterization of the role of metabolism in AD etiology using large-scale metabolomics could contribute to comprehensive understanding of $\mathrm{AD}$ pathology and discovery of mechanisms triggering symptom occurrence [13]. A previous study showed that altered plasma arginine metabolism preceded behavioral and brain metabolic profile changes in APP/PS1 transgenic mice [14]. Several studies have reported alterations in phospholipids, amine metabolites, and amino acids in the blood of AD patients [7, 12, 15, 16]. However, most of these studies were based on relatively small sample size, and the results were not validated, which makes comparative assessments of these studies difficult [17]. Moreover, traditional metabolomics-based studies of AD have mainly relied on comparisons between patients with $\mathrm{AD} /$ mild cognitive impairment (MCI) and healthy controls, and ignored potential interference from other neurological diseases, which has resulted in difficulty in determination of specificity of potential biomarkers.

In this study, we used a robust ultra-performance liquid chromatography-mass spectrometry (UPLC-MS)based untargeted metabolomics approach to profile the plasma metabolome in $\mathrm{AD}$. By comparing the metabolic differences between patients with $\mathrm{AD}$ and cognitively normal controls (Ctrl) using biostatistical and bioinformatics approaches, we highlighted specific biochemical pathways that were perturbed in AD. To further validate our findings and identify promising biomarker candidates, we repeated our analysis in an independent cohort that included patients with AD, MCI, Ctrl, and non-AD neurological disease controls (NDC). We developed a predictive model capable of distinguishing $\mathrm{AD}$ from Ctrl and NDC subjects and provided a novel perspective for early risk assessment of $\mathrm{AD}$ (Fig. 1).

\section{Schematic diagram of study design}

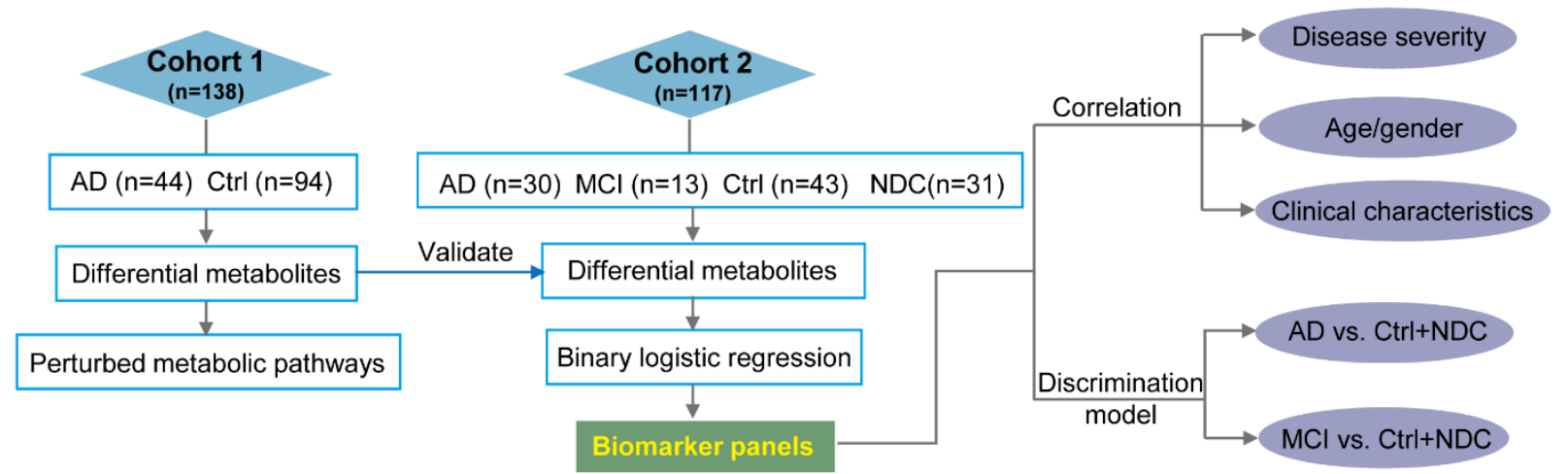

Figure 1. Experimental design. An overview workflow of the metabolomics analysis in Alzheimer's disease. A total of 255 plasma samples were collected and subjected to untargeted metabolomics analysis. Amony them, 138 and 117 plasma samples were included in discovery phase and validation phase, respectively.

\section{MATERIALS AND METHODS}

Study cohorts and patients
Fasting plasma samples were collected at the First Affiliated Hospital of Dalian Medical University. Two hundred fifty-five plasma samples were collected, and all 
subjects or their legally authorized caregivers provided written informed consent. In the discovery phase, we collected 138 plasma samples, of which 44 were from patients with $\mathrm{AD}$ and 94 were from Ctrl subjects. These samples were selected solely based on sample availability. In the validation phase, 117 plasma samples were collected from patients with AD $(\mathrm{n}=30)$, Ctrl subjects ( $\mathrm{n}$ $=43)$, NDC subjects $(n=31 ; 10$ seizure, 10 migraine, 9 peripheral neuropathy, and 2 essential tremor), and patients with MCI $(n=13)$. Patients with $A D$ and patients with MCI were subjected to standard cognitive screening including medical history assessment, physical, cognitive (Mini-mental State Examination (MMSE) scale and
Montreal Cognitive Assessment (MoCA) scale) and neurologic examinations, routine blood and urine tests, and magnetic resonance imaging. $\mathrm{AD}$ diagnoses were made based on the National Institute of Neurological and Communicative Disorders and Stroke-Alzheimer's Disease and Related Disorders Association criteria [18], and MCI was diagnosed using the criteria of Petersen [19, 20]. Patients with non-AD neurological diseases were diagnosed by experienced physicians from the Department of Neurology. This study was approved by the Ethics Committee of the First Affiliated Hospital of Dalian Medical University. Patient characteristics are presented in Table 1.

Table 1. Clinical information of the subjects in the study.

\begin{tabular}{|c|c|c|c|c|c|}
\hline & $\begin{array}{c}\text { AD } \\
(n=74)\end{array}$ & $\begin{array}{c}\text { Ctrl } \\
(n=137)\end{array}$ & $\begin{array}{c}\text { NDC } \\
(n=31)\end{array}$ & $\begin{array}{c}\text { MCI } \\
(n=13)\end{array}$ & $p$ \\
\hline \multicolumn{6}{|l|}{ Cohort 1} \\
\hline Number of individuals & 44 & 94 & - & - & - \\
\hline Age, mean \pm SE & $72.0 \pm 1.3$ & $68.6 \pm 0.8$ & - & - & $0.0156^{\mathrm{a}}$ \\
\hline Gender $(\mathrm{m} / \mathrm{f})$ & $20 / 24$ & $51 / 43$ & - & - & $0.3350^{\mathrm{b}}$ \\
\hline Duration of disease (year) ${ }^{c}$, mean $\pm \mathrm{SE}$ & $4.0 \pm 0.5$ & - & - & - & - \\
\hline $\begin{array}{l}\text { Disease progression } \\
\text { early/middle/late stage }\end{array}$ & $17 / 10 / 3$ & - & - & - & - \\
\hline $\mathrm{MMSE}$, mean $\pm \mathrm{SE}^{\mathrm{e}}$ & $15.2 \pm 1.0$ & - & - & - & - \\
\hline $\mathrm{MoCA}$, mean $\pm \mathrm{SE}^{\mathrm{f}}$ & $10.9 \pm 0.8$ & - & - & - & - \\
\hline \multicolumn{6}{|l|}{ Cohort 2} \\
\hline Number of individuals & 30 & 43 & 31 & 13 & - \\
\hline Age, mean \pm SE & $71.6 \pm 1.6$ & $65.5 \pm 1.2$ & $62.2 \pm 1.8$ & $67.9 \pm 2.0$ & 0.0011 \\
\hline Gender $(\mathrm{m} / \mathrm{f})$ & $10 / 20$ & $25 / 18$ & $17 / 14$ & $8 / 5$ & 0.1459 \\
\hline Duration of disease (year), mean $\pm \mathrm{SE}$ & $4.5 \pm 0.5$ & - & - & $3.9 \pm 0.8$ & $0.5852^{\mathrm{g}}$ \\
\hline $\begin{array}{l}\text { Disease progression } \\
\text { early/middle/late stage }\end{array}$ & $14 / 9 / 4$ & - & - & - & - \\
\hline MMSE, mean \pm SE & $15.4 \pm 1.5$ & - & - & $25.1 \pm 1.0$ & $<0.0001$ \\
\hline MoCA, mean \pm SE & $10.8 \pm 1.5$ & & & $20.0 \pm 1.5$ & 0.0003 \\
\hline \multicolumn{6}{|c|}{$\begin{array}{l}\text { a: Student t-test and one-way ANOVA were applied to calculate the statistical significance of age between AD and Ctrl in cohort } 1 \text { as well as AD, } \\
\text { Ctrl, NDC and MCI in cohort 2, respectively. Notably, there is no significant difference among Ctrl, NDC and MCI with a } p \text { value of } 0.1134 \\
\text { calculated by one-way ANOVA. b: Chi-square test was used to calculate the significant difference of gender composition between groups. c: Three } \\
\text { AD patients in cohort } 1 \text { and one AD patient, one MCI patient in cohort } 2 \text { were lack of information of disease duration. d: Information of disease } \\
\text { progression were not available for fourteen AD patients in cohort } 1 \text { and three AD patients in cohort 2. e, f: Twelve AD patients in cohort } 1 \text { and ten } \\
\text { AD patients, four MCI patients in cohort } 2 \text { were lack of MMSE and MoCA data. g: Student t-test was applied to calculate the statistical significance } \\
\text { of duration of disease, MMSE and MoCA between AD and MCI. AD, Alzheimer's disease; Ctrl, cognitively normal controls; MCI, mild cognitive } \\
\text { impairment; NDC, non-AD neurological diseases controls; MMSE, Mini-mental State Examination; MoCA, Montreal Cognitive Assessment. }\end{array}$} \\
\hline
\end{tabular}

\section{Preparation of plasma samples}

Blood samples were collected in ethylenediaminetetraacetic acid-containing vacutainer tubes by direct venipuncture, then immediately centrifugated at $3,000 \mathrm{rpm}$ for $5 \mathrm{~min}$. Plasma was transferred to sterile tubes, stored at $-80^{\circ} \mathrm{C}$, and thawed on ice prior to analysis. Plasma metabolites were extracted as previously described [21, 22]. One hundred-thirty microliters of plasma was deproteinated using $520 \mu \mathrm{L}$ of methanol that contained 14 internal standards (carnitine C2:0-d3, carnitine C10:0-d3, carnitine C16:0-d3, lysophosphatidylcholine (LPC) 12:0, leucine-d3, phenylalanine-d5, tryptophan-d5, cholic acid-d4, chenodeoxycholic acid-d4, choine-d4, palmitic acid-d3, stearic acid-d3, succinic acid-d4, and tridecanoic acid), then vortexed for $1 \mathrm{~min}$. After centrifugation for $15 \mathrm{~min}\left(14,000 \mathrm{~g}, 4^{\circ} \mathrm{C}\right)$, the supernatant was divided into two aliquots, transferred to fresh tubes, then dried in a vacuum centrifuge. Prior to LC-MS analysis, the dried powder was reconstituted in 65 $\mu \mathrm{L}$ of $25 \%(\mathrm{v} / \mathrm{v})$ aqueous methanol.

A biological quality control (QC) sample was made by pooling $10-20 \mu \mathrm{L}$ of each plasma sample. QC samples were prepared in parallel with study samples and analyzed after 10 sample injections to monitor the robustness of the large-scale analysis. 

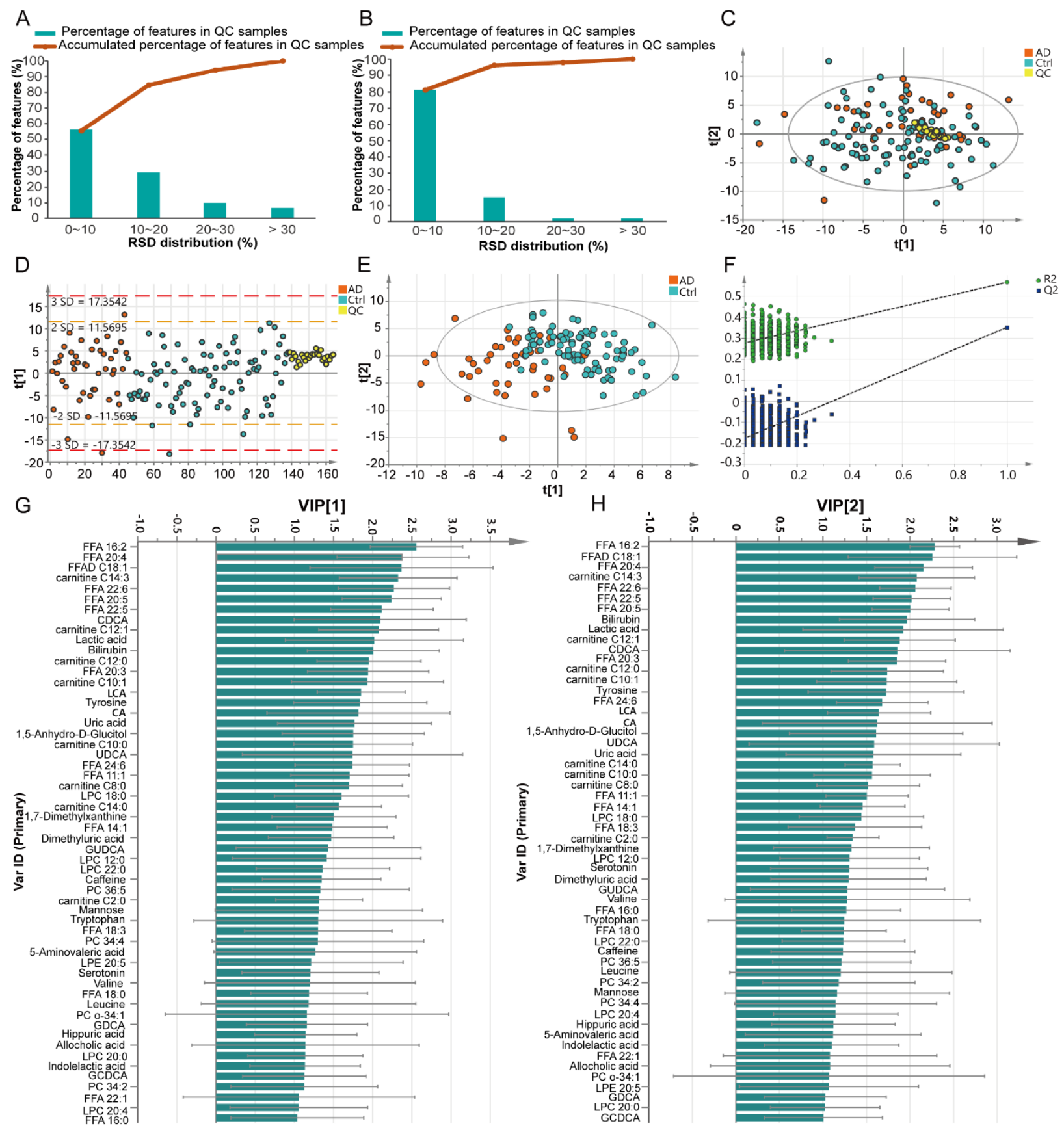

Figure 2. Quality control of the analytical method and multivariate statistical analysis. (A) RSD distribution of all the detected features in the QC samples. (B) RSD distribution of the identified metabolites in the QC samples. (C) PCA score plot. QC samples clustered tightly on the plot, indicating good quality control of the analytical method. (D) Standard deviation of the samples. Most of the samples were located within \pm 2 SD. (E) Score plot of the PLS-DA model. Model parameters: R2Y=0.575, Q2=0.427. (F) Permutation test of the PLS-DA model. 999 permutations resulted in intercepts of R2 $=0.266, \mathrm{Q} 2=-0.175$, indicating an acceptable model without overfitting. (G) Metabolites with VIP [1] score > 1 in PLS-DA model. (H) Metabolites with VIP [2] score > 1 in PLSDA model.

\section{Metabolomic profiling}

Untargeted metabolomic profiling was performed using UPLC (Waters Corporation, Manchester, UK) coupled to a tripleTOF ${ }^{\mathrm{TM}} 5600$ plus (Applied Biosystems, Foster City, CA) mass spectrometry system. For electrospray ionization positive (ESI+) mode detection, an ACQUITY UPLC BEH C8 $1.7 \mu \mathrm{m}$ column was used. The mobile 
phases were $0.1 \%$ formic acid in water and acetonitrile. For ESI negative (ESI-) mode detection, an ACQUITY UPLC HSS T3 $1.8 \mu \mathrm{m}$ column was used. The mobile phases were $6.5 \mathrm{mM} \mathrm{NH} \mathrm{NHCO}_{3}$ in water and $95 \%$ methanol (v/v) with $6.5 \mathrm{mM} \mathrm{NH} 4 \mathrm{HCO}_{3}$. The gradient elution profile and mass spectrometry parameters were published in our previous studies [21, 23].

\section{Metabolite recognition and data preprocessing}

Metabolite identification was performed using an inhouse database that includes about 2,000 metabolites [24]. QC samples were used to generate qualitative information including retention time, accurate mass $(\mathrm{m} / \mathrm{z})$ and MS2 spectrum by performing automatic MS2 scans in information-dependent acquisition mode. Retention time calibration, precursor ion and auto-MS2 information matching were performed using OSI/SMMS software. Peak detection and alignment were conducted using Marker View software (version 1.2.1.1, Applied Biosystems). After refining peaks using the $80 \%$ rule [25], the original peak area of each retained peak was calibrated by internal standards prior to statistical analysis. For each peak, the internal standard was selected to obtain the minimum relative standard deviation (RSD) for the peak in the QC samples. For metabolites detected and identified in the discovery and validation phases, the original peak area was calibrated using the same internal standards.

\section{Statistical analysis}

Multivariate analyses were performed using SIMCA 13.0 (Umetrics AB, Umea, Sweden). An unsupervised model of principal component analysis (PCA) was used to assess the stability of the analytical process and to visualize global metabolome differences among the groups. To maximize class discrimination necessary for discovery of potential metabolic biomarkers between the $\mathrm{AD}$ and Ctrl groups, a supervised model of partial least squares discriminant analysis (PLS-DA) was applied. The variable importance in the projection (VIP) value was used to estimate the discriminatory power of each variable for separation of groups in the PLS-DA model, and variables with VIP values $>1$ were considered important. Furthermore, we performed a permutation test to ensure that the PLS-DA model was not overfitting.

Univariate analyses utilized Student's t-test $(p<$ 0.05). A heat map was constructed using Multi Experiment Viewer (Version 4.7.4) software to visualize the concentrations of metabolites in different groups. To determine the metabolic pathway changes relevant to the $\mathrm{AD}$ disease process, we performed pathway enrichment analysis using the online software MetaboAnalyst (www.metaboanalyst.ca). Binary logistic regression analysis was applied to establish a potential metabolite panel, and a summary receiver operating characteristic (ROC) curve was generated to evaluate the predictive performance of the model. Spearman correlation was used to determine associations between categorical variables (disease severity/gender) and levels of metabolites. Pearson correlation was used to determine associations among continuous variables (age/clinical characteristics) using SPSS 18.0 software (SPSS, Inc., USA).

\section{RESULTS}

To define differential metabolites and perturbed metabolic pathways that differentiated $\mathrm{AD}$ from neurologically normal controls, we performed an untargeted metabolomics approach using plasma samples from $44 \mathrm{AD}$ patients and $94 \mathrm{Ctrl}$ individuals. Totally, 2,412 ion signatures in ESI+ mode and 2,672 ion signatures in ESI- mode were detected in both $\mathrm{AD}$ and Ctrl groups. Using our in-house database, 208 metabolites were identified. The RSD of QC samples was used as an indicator of analytical reproducibility. The results showed that $93.8 \%$ of all detected peaks had RSD values $<30 \%$ (Fig. 2A), and $98.1 \%$ of the identified peaks had RSD values $<30 \%$ (Fig. 2B), which indicated that the analysis was repeatable and robust. The 208 identified ion signatures were further analyzed using an unsupervised, multivariate classification technique. QC samples clustered tightly on the PCA score plot (Fig. 2C), and most samples $(98.2 \%, 161 / 164)$ were within the $95 \%$ confidence interval (CI) in the direction of the first principal component (Fig. 2D), which further confirmed the reliability of this study.

\section{Discovery of differential metabolites and dysregulation of metabolic pathways in $A D$}

To visualize differences within and between groups, and to determine important metabolites that could be used to distinguish $\mathrm{AD}$ and Ctrl, a PLS-DA model with unit variance scaling was established. The plasma metabolomes of $\mathrm{AD}$ and Ctrl were significantly different (Fig. 2E). The model was confirmed to not be overfitted following 999 permutation tests (Fig. 2F). Among the 208 identified metabolites, 56 metabolites were found to be important to the separation of AD and Ctrl with both VIP [1] and VIP [2] values > 1 (Fig. 2G-2H). These 56 metabolites were subjected to univariate analysis (Student's t-test), and 52 of them were found to be statistically significant $(p<0.05)$. The levels of these 52 metabolites were visualized in a heat map (Fig. 3A). Because of the age imbalance between the Ctrl and AD group, we further investigated correlations between levels of metabolites and age. Of the 52 differential metabolites, 
only phosphatidylcholine (PC) O-34:1 showed a significant correlation with age in the Ctrl group $(p=$ $0.0013, r=0.327)$. Therefore, the differences in metabolic levels between $\mathrm{AD}$ and Ctrl were not owing to age difference between the two groups. Most of the differential metabolites, including acylcarnitines, fatty acids (FAs), several LPCs (12:0, 18:0, 20:0, 22:0) and PCs (C34:4 and C36:5), amino acids (valine, tryptophan), serotonin, and several organic acids, were reduced in AD. In contrast, bile acids including allocholic acid, cholic acid (CA), chenodeoxycholic acid (CDCA), glycolchenodeoxycholic acid (GDCA), ursodeoxycholic acid (UDCA), glycochenodeoxycholic acid (GCDCA), and glycoursodeoxycholic acid (GUDCA), and lactic acid, leucine, and tyrosine were elevated in AD. Furthermore, pathway enrichment analysis showed that alpha linolenic acid and linoleic acid metabolism, bile acid biosynthesis, caffeine metabolism, thyroid hormone synthesis, and catecholamine biosynthesis were the most significantly perturbed pathways in AD (Fig. 3B).
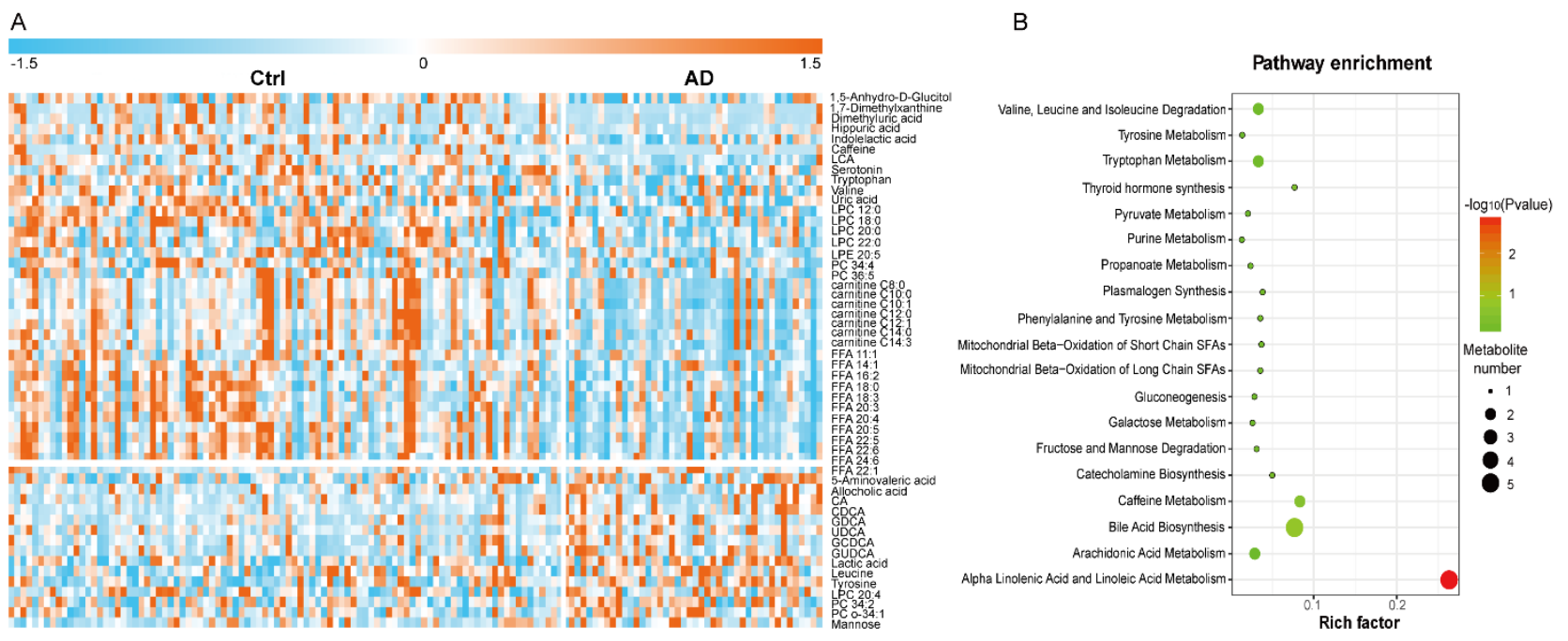

Figure 3. Differential metabolites and perturbed metabolic pathways in AD. (A) Heat map of the significantly changed metabolites in AD compared with Ctrl. (B) Pathway enrichment analysis based on differential metabolites.

Differential metabolites validation and metabolite panel establishment

To further validate the specificity of these differential metabolites in $\mathrm{AD}$, we collected and analyzed an independent cohort of plasma samples from $30 \mathrm{AD}, 43$ Ctrl, $31 \mathrm{NDC}$, and $13 \mathrm{MCI}$ cases. In the validation phase, we observed markedly lower levels of FAs and acylcarnitines in AD compared to those in the Ctrl group, which was consistent with previous results. However, we found that patients in the NDC group showed a greater decrease in the levels of FAs and acyl-carnitines, which indicated that changes in these metabolites were not specific (Fig. 4A). Following comparisons between AD and Ctrl, as well as AD and NDC, levels of five metabolites were found to be specifically altered in $\mathrm{AD}$. The levels of CA, CDCA, and allocholic acid were increased, while the levels of indolelactic acid and tryptophan were decreased in AD compared with those in the Ctrl and NDC groups (Fig. 4B-4F).

ROC curves of classification models based on the 5 specific metabolites were plotted to distinguish $\mathrm{AD}$ from control groups. The area-under-the-curve (AUC) values for each metabolite were $0.721,0.700,0.691,0.643$, and 0.632 , respectively. The discriminating power was improved by combining these 5 metabolites into a metabolite panel using binary logistic regression analysis, as evidenced by an AUC value of 0.822 . Because of the imbalance in age distribution between $\mathrm{AD}$ and the other three groups (Ctrl, MCI and NDC), we further analyzed the correlations between the selected metabolites and age in each group. We found that none of the five metabolites showed significant correlations with age in NDC or MCI, whereas the levels of CA, CDCA, allocholic acid, and tryptophan were significantly associated with age in $\mathrm{AD}$ (Fig. 4G-4V). Therefore, we also included age as a covariate for development of a binary logistic regression model. The corresponding ROC curve showed an AUC value of 0.840 with $76.7 \%$ sensitivity and $83.3 \%$ specificity (Fig. 4W). Moreover, this metabolite panel also showed satisfactory diagnostic performance for distinguishing AD from both Ctrl and NDC with AUC, sensitivity, and specificity of $0.831,86.5 \%$, and $70.0 \%$, respectively (Fig. 4X). Notably, we found that changes in bile acids were more significant in patients MCI (Fig. 4B4D). Using CDCA alone to differentiate MCI from Ctrl 
resulted in an AUC value of 0.850 with a 95\% CI of 0.721- from both Ctrl and NDC groups with a 95\% CI of 0.7050.979 (Fig. 4Y). Furthermore, CDCA alone produced a 0.973 (Fig. 4Z). The discriminating power of each bile satisfactory AUC value of 0.839 for differentiating MCI acid metabolite was presented in Table 2.

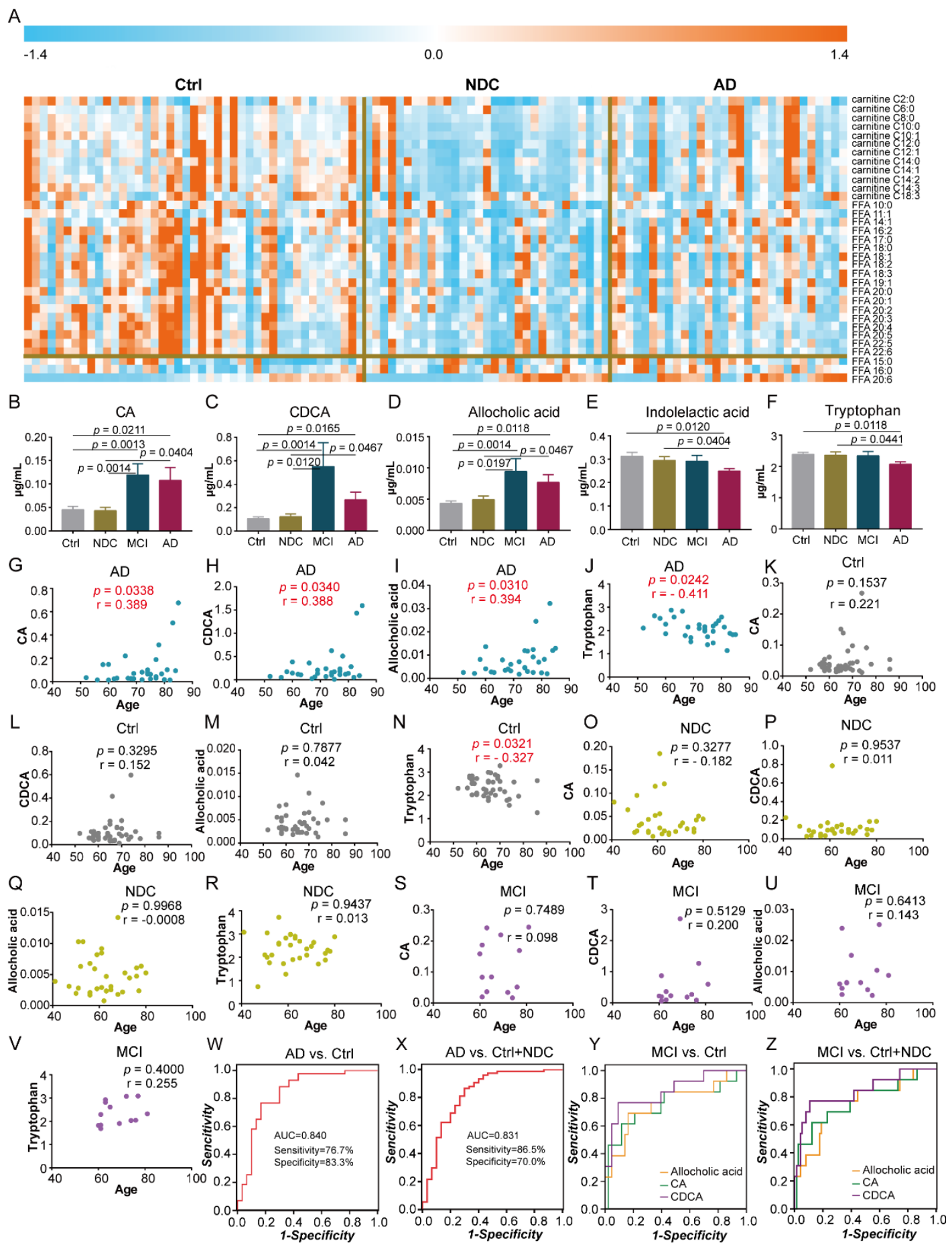

Figure 4. Metabolite panel and ROC analysis. (A) Heat map of FAs and acyl-carnitines in Ctrl, NDC and AD. (B) (F) Bar graphs of the 5 metabolites in different groups. The $p$ values were adjusted for multiple testing using BenjaminiHochberg method. (G) (V) Correlation analysis of the five metabolites with age in AD, Ctrl, NDC and MCI. (W) (X) ROC analysis of the metabolite panels to discriminate AD from Ctrl/ (Ctrl +NDC). (Y) (Z) ROC analysis of bile acids to discriminate $\mathrm{MCI}$ from $\mathrm{Ctrl} /(\mathrm{Ctrl}+\mathrm{NDC})$. 
Table 2. Results for assessment of plasma metabolite panel in the discrimination of AD/MCI.

\begin{tabular}{cccccccc}
\hline & Metabolite panel & AUC & 95\% CI & Sensitivity & Specificity & SE & Significance \\
\hline \multirow{2}{*}{ Meta+age } & AD vs. Ctrl & 0.840 & $0.740 \sim 0.941$ & $76.7 \%$ & $83.3 \%$ & 0.0512 & $8.57 \mathrm{E}-07$ \\
Allocholic & AD vs. (Ctrl+NDC) & 0.831 & $0.733 \sim 0.928$ & $86.5 \%$ & $70.0 \%$ & 0.0496 & $1.39 \mathrm{E}-07$ \\
acid & MCI vs. Ctrl & 0.766 & $0.604 \sim 0.928$ & $69.2 \%$ & $83.7 \%$ & 0.0827 & $3.95 \mathrm{E}-03$ \\
\multirow{2}{*}{ CA } & MCI vs. (Ctrl+NDC) & 0.745 & $0.591 \sim 0.900$ & $69.2 \%$ & $77.0 \%$ & 0.0787 & $4.96 \mathrm{E}-03$ \\
& MCI vs. Ctrl & 0.758 & $0.585 \sim 0.932$ & $61.5 \%$ & $88.4 \%$ & 0.0887 & $5.04 \mathrm{E}-03$ \\
\multirow{2}{*}{ CDCA } & MCI vs. (Ctrl+NDC) & 0.770 & $0.612 \sim 0.928$ & $61.5 \%$ & $87.8 \%$ & 0.0807 & $1.96 \mathrm{E}-03$ \\
& MCI vs. Ctrl & 0.850 & $0.721 \sim 0.979$ & $76.9 \%$ & $90.7 \%$ & 0.0659 & $1.48 \mathrm{E}-04$ \\
& MCI vs. (Ctrl+NDC) & 0.839 & $0.705 \sim 0.973$ & $76.9 \%$ & $89.2 \%$ & 0.0684 & $1.04 \mathrm{E}-04$ \\
\hline
\end{tabular}

$\mathrm{CI}$, confidence interval; SE, standard error.

Associations of metabolites with gender, disease severity, and clinical characteristics

No significant correlations were found between the five selected metabolites and gender (Fig. 5A-5E). Interestingly, the levels of CDCA and allocholic acid were positively correlated with disease severity of $\mathrm{AD}$, and the levels of indolelactic acid and tryptophan were negatively correlated with disease severity (Fig. 5F-5K). Furthermore, correlation analyses of the levels of metabolites with clinical characteristics were performed to characterize the relationship between plasma metabolic profiles and the clinical phenotype of $\mathrm{AD}$. We found that the levels of CA and CDCA were positively correlated with total bile acids (TBA), and the levels of allocholic acid were positively correlated with $\gamma$-glutamyltransferase $(\gamma-\mathrm{GT})$. Furthermore, indolelactic acid was positively correlated with uric acid (UA), while tryptophan levels were positively correlated with $\gamma$-GT and alanine aminotransferase (ALT), and negatively correlated with free thyroxine 4 (FT4) (Fig. 5L). Associations between the selected metabolites and MMSE and MoCA scores were also investigated in AD patients. However, no significant correlations were observed (Supplementary Table 1).

\section{DISCUSSION}

In this study, we employed untargeted metabolomics and biostatistical approaches to gain further insight into metabolic network failures and to identify potential metabolic biomarkers in $\mathrm{AD}$. A total of 255 plasma samples (AD, $\mathrm{n}=74$; MCI, $\mathrm{n}=13$; Ctrl, $\mathrm{n}=137$; NDC, $\mathrm{n}$ $=31$ ) were collected and analyzed. First, we compared the plasma metabolomes of $\mathrm{AD}$ patients with matched cognitively normal individuals. Our results suggested that the overall metabolic expression profile differed between the two groups. Following VIP value filtering and multivariate and univariate analyses, 52 metabolites were found to be differentially altered in AD. Pathway enrichment analysis showed that alpha linolenic acid, linoleic acid, and arachidonic acid metabolism, mitochondrial beta-oxidation of saturated FAs, bile acid biosynthesis, and amino acid metabolism were the most relevant pathways associated with $\mathrm{AD}$.

\section{Metabolic pathway dysregulations in AD}

We observed marked reductions in the levels of FAs (except for C22:1). In particular, polyunsaturated fatty acids (PUFAs) levels were reduced in AD compared with those in Ctrl, which agreed with previous studies [26]. PUFAs are neuroprotective, and decreased levels of PUFAs may contribute to the cognitive decline associated with $\mathrm{AD}$ [27]. A recent study showed that unsaturated FA (UFA) metabolism was perturbed in the brains of $\mathrm{AD}$ patients. This study showed significant associations between UFA levels and neuritic plaques, neurofibrillary tangle burden, and cognitive performance [13]. Docosahexaenoic acid (DHA, C22:6) is the PUFA most associated with $\mathrm{AD}$. Our results showed a reduction in DHA biosynthesis in $\mathrm{AD}$, which corroborated the results from previous studies that reduced levels of DHA may be resulted from defective FA metabolism in the liver [27, 28]. Dietary supplementation with DHA has been shown to improve cognitive performance in several animal models of $\mathrm{AD}$ and in $\mathrm{AD}$ patients [29, 30]. PUFAs also play important roles in $\mathrm{AD}$ pathology. In vivo studies suggested that linoleic acid (C18:2), arachidonic acid (C20:4), and oleic acid (C18:1) induced polymerization of tau and $\mathrm{A} \beta$ and contributed to $\mathrm{AD}$ pathological progress $[13,31]$.

Additionally, we also noted that levels of nearly all acyl-carnitines were lower in AD than those in the Ctrl group, but only medium- and long-chain acyl-carnitines were significantly different between the two groups. A previous study indicated that serum acetyl-carnitine and other acyl-carnitine levels declined across the continuum from healthy individuals to subjects with subjective memory impairment and MCI, and patients with $\mathrm{AD}$, which agreed with our findings [2]. Deficits in mediumand long-chain acyl-carnitines indicated disruption of FA transport into the mitochondria for beta-oxidation, and impaired energy metabolism [2]. These results also can be 
evidenced by the findings from proteomic and transcriptomic studies, which showed decreased carnitine shuttle activity and FA beta-oxidation in AD patients [32, 33]. Significant reductions in the levels of acyl carnitines were observed in the hippocampus and cerebral cortex of
APP/PS1 transgenic mice [34]. Deficits in carnitine shuttling and beta-oxidation might be associated with mitochondrial dysfunction that contributed to the pathogenesis of $\mathrm{AD}$.
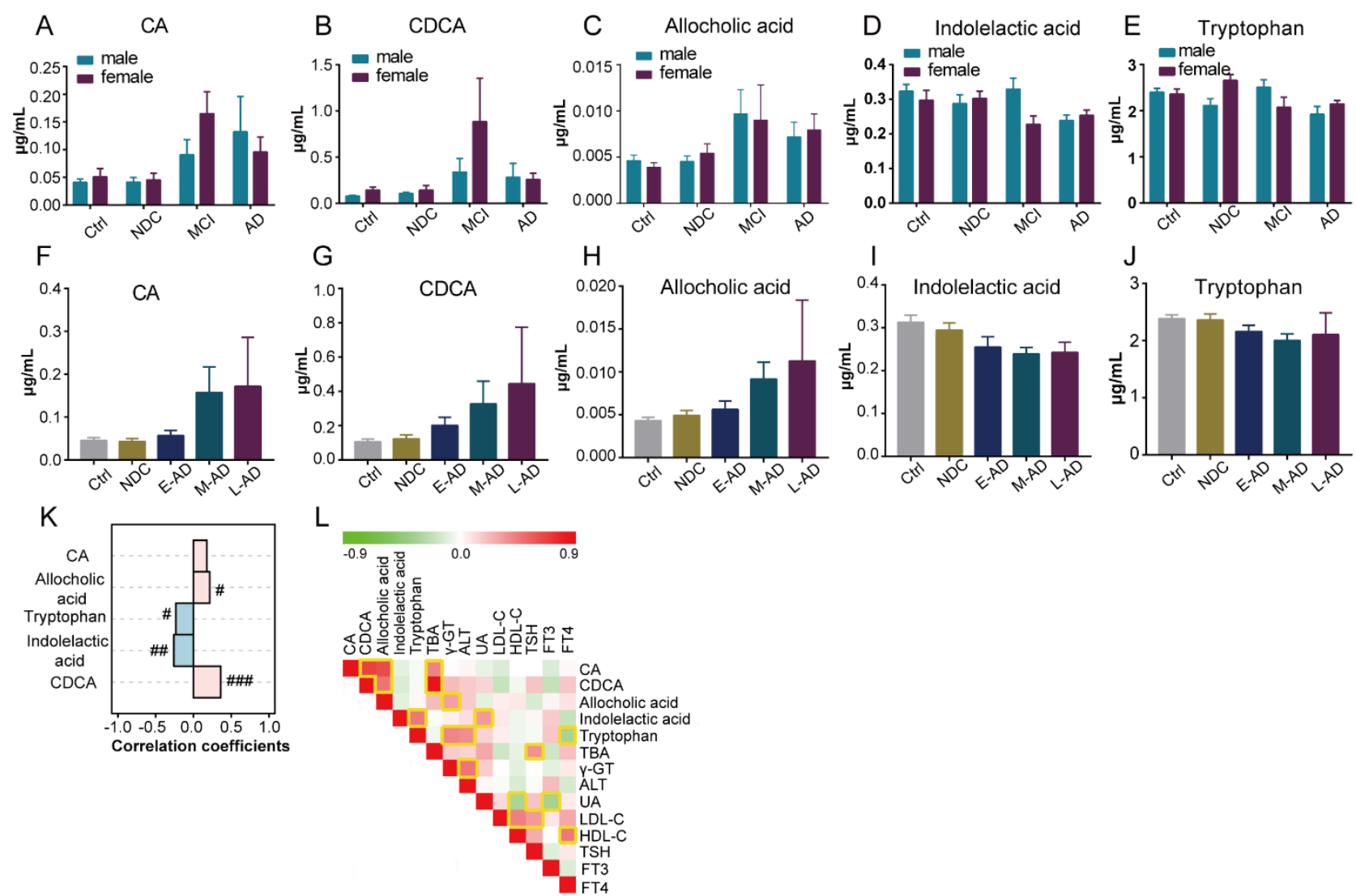

Figure 5. Correlation analysis of the metabolites panel with gender, disease severity and clinical characteristics. (A) (E) Distribution of the levels of metabolite in different genders in Ctrl, NDC, MCI and AD. (F) (K) Correlation analysis of the metabolite levels to disease progress. Correlation coefficients were based on Spearman correlation analysis. \#, $0.01<p<0.05$, \#\#, $0.001<p<0.01$, \#\#\#, $0.0001<p<0.001$. (L) Correlations analysis of metabolite levels with clinical parameters, which was based on Pearson correlation analysis. Yellow square indicated the correlations between two variables were statistically significant.

In contrast to the decreased levels of FAs and acylcarnitines, we observed increased levels of bile acids in $\mathrm{AD}$, with the exception of lithocholic acid (LCA). The levels of CA, CDCA, and allocholic acid were increased with disease severity. Elevated levels of bile acids in the circulation usually result from increased bile acid synthesis and liver dysfunction [35], which was consistent with our findings of significant correlations between CA, CDCA and TBA and between allocholic acid and $\gamma-\mathrm{GT}$. Recently, a larger multi-center study from the Alzheimer's Disease Neuroimaging Initiative cohort used targeted metabolomic profiling, and reported strong associations between bile acid profiles and $A \beta$, tau, and neurodegeneration biomarkers, which suggested that the gut-liver-brain axis may play a role in $\mathrm{AD}$ pathogenesis
$[1,36]$. Primary bile acids are initially synthesized from cholesterol in the liver, and are converted to secondary bile acids by intestinal anaerobic bacteria [1]. In addition to potential effects from diet and cholesterol metabolism, conditions such as dysbiosis and antibiotic treatment might cause variations in bile acids levels [37]. Our study further supported a strong association between $A D$ and abnormal bile acid metabolism. Further investigation of the underlying contributions of bile acids to the onset and progression of $\mathrm{AD}$ may result in a novel hypothesis of $\mathrm{AD}$ pathogenesis.

Perturbations in amino acid metabolism were also observed in our study. Reduced levels of tryptophan, serotonin, and indolelactic acid suggested that tryptophan degradation was enhanced through the kynurenine 
pathway in AD. Tryptophan is a precursor of several bioactive compounds including neurotransmitters (e.g., serotonin), and its metabolism plays an important role in regulating neuronal activity and the inflammatory response [38]. Furthermore, lower plasma tryptophan levels were associated with decreased olfactory function in elderly populations [38]. We observed a continuous decrease in levels of tryptophan and indolelactic acid with the progression of $\mathrm{AD}$. In addition, tryptophan showed an age-related decrease in both $\mathrm{AD}$ and Ctrl groups, and significant correlations with $\gamma$-GT, ALT, and FT4 in our study, which suggested that tryptophan may be a clinical marker of disease risk in the elderly. Recently, an in vitro study showed that tryptophan, serotonin, melatonin, and other indole compounds, protected against $A \beta$ peptide aggregation and cytotoxicity [39]. Investigation of the possible mechanism underlying the neuroprotective effects of indole compounds may result in identification of novel potential therapeutic targets for AD.

\section{Metabolite panels for $A D$ discrimination}

To screen stable and specific biomarkers for $\mathrm{AD}$ discrimination, we repeated our analysis in an independent cohort that included $\mathrm{AD}, \mathrm{Ctrl}, \mathrm{MCI}$, and NDC groups to further validate previous findings. Inclusion of the NDC group as a disease control was intended to provide an additional control for evaluation of biomarker specificity. According to our results, reduced levels of FAs and acyl-carnitines in the AD and NDC groups limited their use as diagnostic biomarkers for AD. We identified a metabolite panel comprised of five metabolites including CA, CDCA, allocholic acid, indolelactic acid, and tryptophan that may allow for discrimination of subjects with $\mathrm{AD}$ from cognitively normal and non-AD neurological disease controls.

In addition to serving as potential diagnostic biomarkers, the five AD-specific markers identified in this study showed significant correlations with disease progression, which suggested that these markers or the pathways from which they derive may be potential targets for treatment of AD. In addition, significant differences in levels of bile acids, particularly CDCA, in MCI suggested that disruption of bile acid profiles may serve as a potential indicator of $\mathrm{AD}$ risk. These results agreed with those of previous studies that demonstrated strong associations between bile acid profiles and cognitive impairment in $\mathrm{AD}[36,40]$.

In summary, combination of metabolomics with statistical and bioinformatic analyses resulted in identification of overall metabolic network failures in AD. Our results indicated that dysregulation of PUFA metabolism, mitochondrial $\beta$-oxidation, bile acid synthesis, and tryptophan metabolism were pivotal events in $\mathrm{AD}$ progression. Further investigation of the specific roles of these metabolic changes in the pathogenesis of AD might result in development of novel therapeutic strategies. However, further studies are needed to validate our findings. Although a validation phase was included in our study, the samples size is still insufficient, and future validation studies should include multi-center clinical trials with larger sample sizes. Furthermore, metabolomics-based longitudinal studies of patients with $\mathrm{AD}$ in the pre-symptomatic phase will be crucial to the identification of novel biomarkers for early diagnosis and early intervention.

\section{Acknowledgements}

This work was supported by National Natural Science Foundation of China (81430021, 81771521), National Key Research and Development Program of China (2017YFC0906900), Key Research \& Development Plan of Liaoning Science and Technology Department (2018225051) and Dalian Medical Science Research Program (1812008). We thank all the participants and their families for their commitments and statements to help advance research into clinical diagnosis of AD.

\section{Conflict of Interest}

The authors declare no competing interests.

\section{Supplementary Materials}

The Supplemenantry data can be found online at: www.aginganddisease.org/EN/10.14336/AD.2020.0217.

\section{References}

[1] MahmoudianDehkordi S, Arnold M, Nho K, Ahmad S, Jia W, Xie G, et al. (2019). Altered bile acid profile associates with cognitive impairment in Alzheimer's disease-An emerging role for gut microbiome. Alzheimers Dement, 15:76-92.

[2] Cristofano A, Sapere N, La Marca G, Angiolillo A, Vitale M, Corbi G, et al. (2016). Serum Levels of Acyl-Carnitines along the Continuum from Normal to Alzheimer's Dementia. PLoS One, 11:e0155694.

[3] Reitz C, Mayeux R (2014). Alzheimer disease: epidemiology, diagnostic criteria, risk factors and biomarkers. Biochem Pharmacol, 88:640-651.

[4] Xu XH, Huang Y, Wang G, Chen SD (2012). Metabolomics: a novel approach to identify potential diagnostic biomarkers and pathogenesis in Alzheimer's disease. Neurosci Bull, 28:641-648.

[5] Bayer AJ (2018). The role of biomarkers and imaging in the clinical diagnosis of dementia. Age Ageing, 47:641-643.

[6] Morgan AR, Touchard S, Leckey C, O'Hagan C, 
Nevado-Holgado AJ, Barkhof F, et al. (2019). Inflammatory biomarkers in Alzheimer's disease plasma. Alzheimers Dement, 15:776-787.

[7] Lin CN, Huang CC, Huang KL, Lin KJ, Yen TC, Kuo HC (2019). A metabolomic approach to identifying biomarkers in blood of Alzheimer's disease. Ann Clin Transl Neurol, 6:537-545.

[8] Startin CM, Ashton NJ, Hamburg S, Hithersay R, Wiseman FK, Mok KY, et al. (2019). Plasma biomarkers for amyloid, tau, and cytokines in Down syndrome and sporadic Alzheimer's disease. Alzheimers Res Ther, 11:26.

[9] Nabers A, Hafermann H, Wiltfang J, Gerwert K (2019). Abeta and tau structure-based biomarkers for a bloodand CSF-based two-step recruitment strategy to identify patients with dementia due to Alzheimer's disease. Alzheimers Dement (Amst), 11:257-263.

[10] Patra K, Soosaipillai A, Sando SB, Lauridsen C, Berge G, Møller I, et al. (2018). Assessment of kallikrein 6 as a cross-sectional and longitudinal biomarker for Alzheimer's disease. Alzheimers Res Ther, 10:9.

[11] Balietti M, Giuli C, Conti F (2018). Peripheral Blood Brain-Derived Neurotrophic Factor as a Biomarker of Alzheimer's Disease: Are There Methodological Biases? Mol Neurobiol, 55:6661-6672.

[12] Tynkkynen J, Chouraki V, van der Lee SJ, Hernesniemi J, Yang Q, Li S, et al. (2018). Association of branched-chain amino acids and other circulating metabolites with risk of incident dementia and Alzheimer's disease: A prospective study in eight cohorts. Alzheimers Dement, 14:723-733.

[13] Snowden SG, Ebshiana AA, Hye A, An Y, Pletnikova $\mathrm{O}$, O'Brien R, et al. (2017). Association between fatty acid metabolism in the brain and Alzheimer disease neuropathology and cognitive performance: A nontargeted metabolomic study. PLoS Med, 14:e1002266.

[14] Bergin DH, Jing Y, Mockett BG, Zhang H, Abraham WC, Liu P (2018). Altered plasma arginine metabolome precedes behavioural and brain arginine metabolomic profile changes in the APPswe/PS1DeltaE9 mouse model of Alzheimer's disease. Transl Psychiatry, 8:108.

[15] Mapstone M, Cheema AK, Fiandaca MS, Zhong X, Mhyre TR, MacArthur LH, et al. (2014). Plasma phospholipids identify antecedent memory impairment in older adults. Nat Med, 20:415-418.

[16] Chouraki V, Preis SR, Yang Q, Beiser A, Li S, Larson MG, et al. (2017). Association of amine biomarkers with incident dementia and Alzheimer's disease in the Framingham Study. Alzheimers Dement, 13:13271336.

[17] Casanova R, Varma S, Simpson B, Kim M, An Y, Saldana S, et al. (2016). Blood metabolite markers of preclinical Alzheimer's disease in two longitudinally followed cohorts of older individuals. Alzheimers Dement, 12:815-822.

[18] Mckhann G, Drachman D, Folstein MF, Katzman R, Price D, Stadlan E (1984). Clinical diagnosis of Alzheimer disease: report of NINCDS-ADRDA Work
Group under the auspices of Department of Health and Human Service Task Force on Alzheimer's Disease. Neurology, 34:939-944.

[19] Petersen RC, Smith GE, Waring SC, Ivnik RJ, Tangalos EG, Kokmen E (1999). Mild Cognitive Impairment: Clinical Characterization and Outcome. Arch Neurol, 56:303-308.

Petersen RC (2004). Mild cognitive impairment as a diagnostic entity. J Intern Med, 256:183-194.

Huang Y, Chen G, Liu X, Shao Y, Gao P, Xin C, et al. (2014). Serum metabolomics study and eicosanoid analysis of childhood atopic dermatitis based on liquid chromatography-mass spectrometry. J Proteome Res, 13:5715-5723.

[22] Luo P, Yin P, Hua R, Tan Y, Li Z, Qiu G, et al. (2018). A Large-scale, multicenter serum metabolite biomarker identification study for the early detection of hepatocellular carcinoma. Hepatology, 67:662-675. [23] Ren S, Shao Y, Zhao X, Hong CS, Wang F, Lu X, et al. (2016). Integration of Metabolomics and Transcriptomics Reveals Major Metabolic Pathways and Potential Biomarker Involved in Prostate Cancer. Mol Cell Proteomics, 15:154-163.

[24] Zhao X, Zeng Z, Chen A, Lu X, Zhao C, Hu C, et al. (2018). Comprehensive Strategy to Construct InHouse Database for Accurate and Batch Identification of Small Molecular Metabolites. Anal Chem, 90:76357643.

[25] Smilde AK, van der Werf MJ, Bijlsma S, van der Werff-van der Vat BJC, Jellema RH (2005). Fusion of mass spectrometry-based metabolomics data. Anal Chem, 77:6729-6736.

[26] Cunnane SC, Schneider JA, Tangney C, TremblayMercier J, Fortier M, Bennett DA, et al. (2012). Plasma and brain fatty acid profiles in mild cognitive impairment and Alzheimer's disease. J Alzheimers Dis, 29:691-697.

[27] Astarita G, Jung KM, Berchtold NC, Nguyen VQ, Gillen DL, Head E, et al. (2010). Deficient liver biosynthesis of docosahexaenoic acid correlates with cognitive impairment in Alzheimer's disease. PLoS One, 5:e12538.

[28] Thomas MH, Paris C, Magnien M, Colin J, Pelleieux $\mathrm{S}$, Coste F, et al. (2017). Dietary arachidonic acid increases deleterious effects of amyloid-beta oligomers on learning abilities and expression of AMPA receptors: putative role of the ACSL4-cPLA2 balance. Alzheimers Res Ther, 9:69.

[29] Swanson D, Block R, Mousa SA (2012). Omega-3 fatty acids EPA and DHA: health benefits throughout life. Adv Nutr, 3:1-7.

[30] Quinn JF, Raman R, Thomas RG, Yurko-Mauro K, Nelson EB, Van Dyck C, et al. (2010). Docosahexaenoic acid supplementation and cognitive decline in Alzheimer disease: a randomized trial. JAMA, 304:1903-1911.

[31] Amtul Z, Uhrig M, Wang L, Rozmahel RF, Beyreuther K (2012). Detrimental effects of arachidonic acid and its metabolites in cellular and mouse models of Alzheimer's disease: structural insight. Neurobiol 
Aging, 33:831.e821-831.e831.

[32] Stempler S, Yizhak K, Ruppin E (2014). Integrating transcriptomics with metabolic modeling predicts biomarkers and drug targets for Alzheimer's disease. PLoS One, 9:e105383.

[33] Dey KK, Wang H, Niu M, Bai B, Wang X, Li Y, et al. (2019). Deep undepleted human serum proteome profiling toward biomarker discovery for Alzheimer's disease. Clin Proteomics, 16:16.

[34] Raúl GD, Tamara GB, Javier V, José Luis GA (2015). Metabolomic screening of regional brain alterations in the APP/PS1 transgenic model of Alzheimer's disease by direct infusion mass spectrometry. J Pharm Biomed Anal, 102:425-435.

[35] Xie G, Wang X, Huang F, Zhao A, Chen W, Yan J, et al. (2016). Dysregulated hepatic bile acids collaboratively promote liver carcinogenesis. Int $\mathrm{J}$ Cancer, 139:1764-1775.

[36] Nho K, Kueider-Paisley A, MahmoudianDehkordi S, Arnold M, Risacher SL, Louie G, et al. (2019). Altered bile acid profile in mild cognitive impairment and
Alzheimer's disease: Relationship to neuroimaging and CSF biomarkers. Alzheimers Dement, 15:232-244. Pan X, Elliott CT, McGuinness B, Passmore P, Kehoe PG, Hölscher C, et al. (2017). Metabolomic Profiling of Bile Acids in Clinical and Experimental Samples of Alzheimer's Disease. Metabolites, 7:28.

[38] Adachi Y, Shimodaira Y, Nakamura H, Imaizumi A, Mori M, Kageyama Y, et al. (2017). Low plasma tryptophan is associated with olfactory function in healthy elderly community dwellers in Japan. BMC Geriatr, 17:239.

[39] Hornedo-Ortega R, Da Costa G, Cerezo AB, Troncoso AM, Richard T, Garcia-Parrilla MC (2018). In Vitro Effects of Serotonin, Melatonin, and Other Related Indole Compounds on Amyloid- $\beta$ Kinetics and Neuroprotection. Mol Nutr Food Res, 62:1700383.

[40] Marksteiner J, Blasko I, Kemmler G, Koal T, Humpel C (2018). Bile acid quantification of 20 plasma metabolites identifies lithocholic acid as a putative biomarker in Alzheimer's disease. Metabolomics, 14:1. 